\title{
Improving outcomes for the treatment of Anal Squamous Cell Carcinoma and Anal Intraepithelial Neoplasia
}

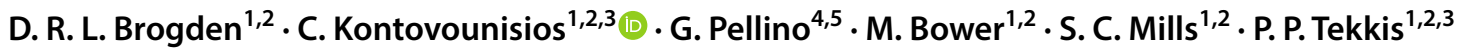

Received: 15 October 2019 / Accepted: 6 November 2019 / Published online: 20 November 2019

(c) Springer Nature Switzerland AG 2019

Anal Squamous Cell Carcinoma (ASCC) is an uncommon cancer, with an incidence rate between 1 and 2 per 100,000 per year. However, its incidence is significantly increasing in established market economies; where incidence rates in men increased between 2.8 and $6.8 \%$ per year and in women $1.5-10.7 \%$ per year between 1971 and 2007 [1].

ASCC has a dysplastic precursor Anal Intraepithelial Neoplasia (AIN) which is associated with oncogenic human papillomavirus (HPV) Infections. Up to $90 \%$ of ASCC tumours have evidence of HPV infection. The understood progression of pathology after a high-risk HPV infection is for patients to progress from low-grade to high-grade AIN over time. If untreated this can then develop into invasive ASCC. The progression of AIN to ASCC is unpredictable, however, and many patients do not progress from the earliest grades of AIN to ASCC without periods of regression.

Risk factors known to predispose to ASCC include smoking, human immunodeficiency virus (HIV), immunosuppression, sexually transmitted infections, receptive anal intercourse as well as concurrent or previous HPV-related genitourinary dysplasia or malignancies.

The reasons behind the rising incidence of ASCC are unclear, one of the suggestions is that it could be related

C. Kontovounisios

c.kontovounisios@imperial.ac.uk

1 Department of Colorectal Surgery, Chelsea and Westminster Hospital, London, UK

2 Department of Surgery and Cancer, Imperial College London, Chelsea and Westminster and the Royal Marsden Campus, London, UK

3 Department of Colorectal Surgery, Royal Marsden Hospital, London, UK

4 Department of Advanced Medical and Surgical Sciences, Università degli Studi della Campania "Luigi Vanvitelli", Naples, Italy

5 Colorectal Unit, University Hospital Vall d'Hebron, Barcelona, Spain to an improvement in life expectancy of patients with HIV who are now developing long-term sequalae of their disease. Indeed, the prevalence of AIN in this patient cohort is extremely high; up to a third of HIV positive men practicing receptive anal intercourse are reported to have high-grade dysplasia. However, up to two-thirds of patients with ASCC are female and it is likely the majority are not HIV positive.

The other suggestion is that changes in sexual behaviour and freedom in developed countries has led to higher numbers of individuals being exposed to oncogenic genitourinary HPV infections. Historically, most patients with ASCC are reported to be women in their 6th decade with previous HPV infections, genitourinary dysplasias and malignancies. However, this is less straightforward when you consider that the incidence of cervical cancer decreased in the same time period. It is possible that the successful treatment of cervical dysplasias within screening programmes may increase this subgroup's risk of ASCC in later life. Although treating cervical dysplasias may prevent a cervical malignancy, it might not resolve an ongoing anogenital oncogenic HPV infection leading to patients surviving a potential early cervical malignancy but later presenting with an ASCC.

The rising incidence of ASCC and its precursor AIN has created a clinical dilemma in establishing best practice. ASCC remains a rare cancer and sample sizes required for meaningful research can be difficult to obtain without significant research collaboration. Large demographic studies are also limited by the data available. Although national cancer databases exist, they are unable to link relative risk factors together. This includes HIV status, anogenital intraepithelial neoplasia and malignancy treatment and outcomes.

As the clinical consensus and practice between geographical areas varies there is little good evidence data on the management of AIN and the prevention of ASCC. Treatments for AIN are available but, so far there is no evidence that their use prevents ASCC. The difficulties of field change relating to oncogenic HPV infections and synchronous highgrade lesions identified after treatment of AIN lesions also 
limit the ability to assess the efficacy of AIN treatments. Unfortunately, unlike cervical screening programmes, there is also little evidence base that High-Resolution Anoscopy programmes can be useful in preventing ASCC. This uncertainty is reflected in current clinical guidelines for the prevention of ASCC [2-4].

Recently there is increasing interest in organ sparing approaches for ASCC, where it may be possible to treat superficially invasive squamous cell carcinomas (SISCCA's) with local excision alone [5]. The PLATO trial is hoping to answer this question in the future. In the meantime, many centers are already trialling local excision with close follow up as the sole treatment for early anal cancers. We feel that the patients most likely to benefit from this approach are high-risk HIV positive patients with a previous AIN diagnosis as they are more likely to present with SISCCA's whilst in surveillance. Recent research into local excision alone in T1 tumours was limited by the inability of existing cancer datasets to include recurrence rates, chemoradiotherapy regimes, treatment complications and important demographic risk factors such as HIV status and prior AIN diagnosis and treatment [5].

We believe that a novel collaborative approach is required to be able to definitively answer questions around the best practice of treating ASCC and AIN. mASCARA (Multinational Anal Squamous Cell Carcinoma Registry and Audit) is the first international research registry for ASCC and highgrade AIN that incorporates HIV, sexual health, AIN and HPV related outcomes not included in standard cancer datasets. It also incorporates management regimes and long term oncological and patient outcomes including recurrence and disease activity, toxicity, survival, incontinence and sexual function.

mASCARA has full ethical approval to use the data stored within the registry for relevant research into AIN and ASCC outcomes. It meets the key 7 General Data Protection Regulation principles allowing research within the European Union and also by providing data sharing agreements, permits countries external to the European Union to participate.

All data is pseudoanonymised to the submitting direct care team and anonymised to researchers to allow direct care teams to update participating patient's data over time without compromising patient confidentiality. mASCARA is hosted on a secure, encrypted, web based, online portal with two-factor authentication. Prospective patients will be required to give their written informed consent prior to inclusion into the registry.

mASCARA launched in September 2019 and began recruiting clinical sites worldwide. Recruited centres will be invited to include retrospective demographics and patient outcomes or to voluntarily include their prospective patients with ASCC or high-grade SCC from the date of recruitment. High-grade AIN is defined as patients with a histological

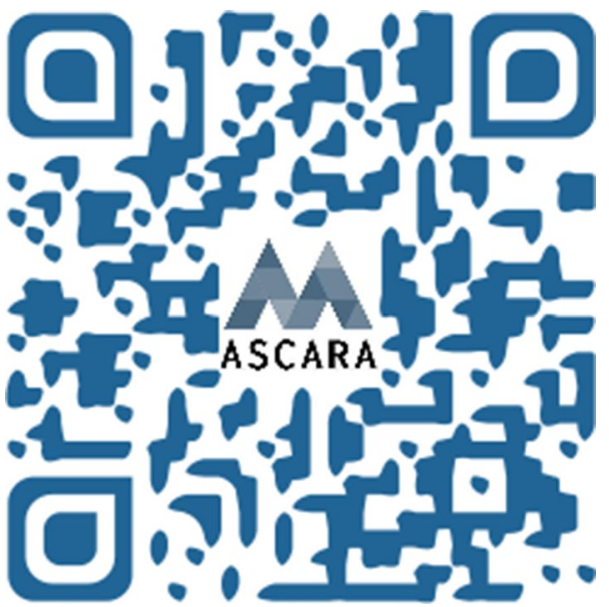

Fig. 1 QR code with link to the mASCARA registry website

diagnosis of high grade squamous intraepithelial lesion (HSIL), high-grade AIN (HGAIN), AIN3 or p16 positive AIN2.

We plan to run the collaborative research registry for 10 years and in that time to answer key research questions regarding demographics, screening, prevention, management and patient outcomes for AIN and ASCC. The registry is open to global participation; information can be found on the dedicated website (http://www.mascararegistry.com/) (Fig. 1).

Our aim is to provide a valuable and meaningful resource for clinicians and researchers interested in ASCC or AIN research.

Funding The development of mASCARA has been funded by a grant from the Red Trouser Day Charity.

\section{Compliance with ethical standards}

Conflict of interest The authors declare that they have no conflict of interest

Ethical approval The present paper was exempt from approval.

Informed consent For this review of literature informed consent was not required.

\section{References}

1. Islami F, Ferlay J, Lortet-Tieulent J, Bray F, Jemal A (2017) International trends in anal cancer incidence rates. Int J Epidemiol 46(3):924-938. https://doi.org/10.1093/ije/dyw276

2. Geh I, Gollins S, Renehan A, Scholefield J, Goh V, Prezzi D, Moran B, Bower M, Alfa-Wali M, Adams R (2017) Association 
of Coloproctology of Great Britain and Ireland (ACPGBI): guidelines for the Management of Cancer of the Colon, Rectum and Anus (2017)—Anal Cancer. Colorectal Dis 19(Suppl 1):82-97. https://doi.org/10.1111/codi.13709

3. Stewart DB, Gaertner WB, Glasgow SC, Herzig DO, Feingold D, Steele SR, Prepared on Behalf of the Clinical Practice Guidelines Committee of the American Society of C, Rectal S (2018) The American society of colon and rectal surgeons clinical practice guidelines for anal squamous cell cancers (revised 2018). Dis Colon Rectum 61(7):755-774. https://doi.org/10.1097/ DCR.0000000000001114

4. Binda GA, Gagliardi G, Dal Conte I, Verra M, Cassoni P, Cavazzoni E, Stocco E, Delmonte S, De Nardi P, Sticchi L, Mistrangelo M (2019) Practice parameters for the diagnosis and treatment of anal intraepithelial neoplasia (AIN) on behalf of the Italian Society of Colorectal Surgery (SICCR). Tech Coloproctol 23(6):513528. https://doi.org/10.1007/s10151-019-02019-5

5. Chai CY, Tran Cao HS, Awad S, Massarweh NN (2018) Management of Stage I squamous cell carcinoma of the anal canal. JAMA Surg 153(3):209-215. https://doi.org/10.1001/jamas urg.2017.3151

Publisher's Note Springer Nature remains neutral with regard to jurisdictional claims in published maps and institutional affiliations. 